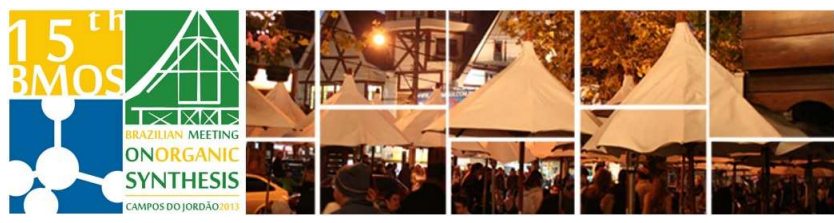

\title{
Synthesis of Simple Alkyl-Seleno-Carbohydrates
}

\author{
Jaqueline P. Vargas (PG) ${ }^{\star 1}$, Diogo S. Lüdtke $(P Q)^{1}$ \\ 1. Instituto de Química, UFRGS, Porto Alegre, RS, Brasil \\ e-mail corresponding author: jaquevgs@gmail.com
}

Keywords: Carbohydrate, Selenium, Neoglycoconjugate

\section{INTRODUCTION}

Selenium exists as trace element in mammalian and their metabolism is still in current investigation. Incorporate the selenium atom in carbohydrates is one way to further this investigations ${ }^{1}$. We planned this work since alkyl glycosides showed antimicrobial activity $^{2}$ and organoselenium serves as important therapeutic compounds, including anticancer agents ${ }^{3}$. Here, we present our work focused in the synthesis of alkyl-seleno linked in non-glycosidally form.

\section{RESULTS AND DISCUSSION}

First of all, the starting materials were synthesized. The alkyl-disselenides 1, by Grignard reaction, using alkyl-bromides as reagents, and the tosylates 2 derivatives of $D$-xylose, $D$-ribose, $D$-galactose from respective protected sugars (figure 1 ).

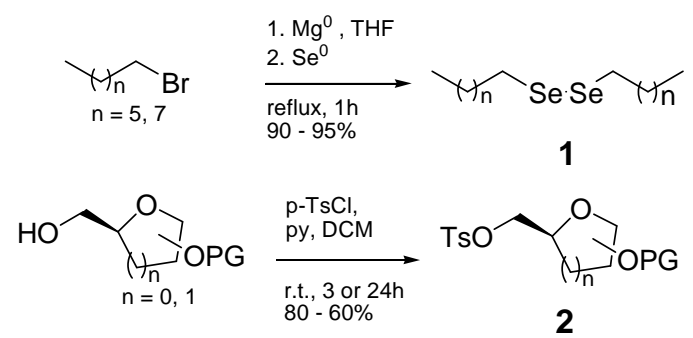

Figure 1. Synthesis of starting materials.

Then, the alkyl-seleno-neoglycoconjugates $\mathbf{3}$ were obtained by reductive cleavage of alkyl-disselenides 1 using $\mathrm{NaBH}_{4}$ as reductant followed by addiction of tosylates $\mathbf{2}^{4}$ (figure 2 ).

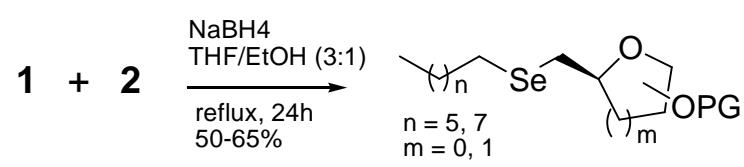

3

Figure 2. Synthesis of alkyl-selenium-glycoconjugates.

The choose for the specific chain of octyl and decyl for the present alkyl-seleno-neoglycoconjugates was based on previous works ${ }^{5}$, which investigate the advantages of obtain $n$-octyl- $\beta$ - $D$-glycosides to study biological activities. Futhermore, the improvement of this work is link the alkyl group in non-glycosidic position through a selenium atom by usual organic synthesis.

Table 1. Alkyl-selenium-neoglycoconjugates scope.

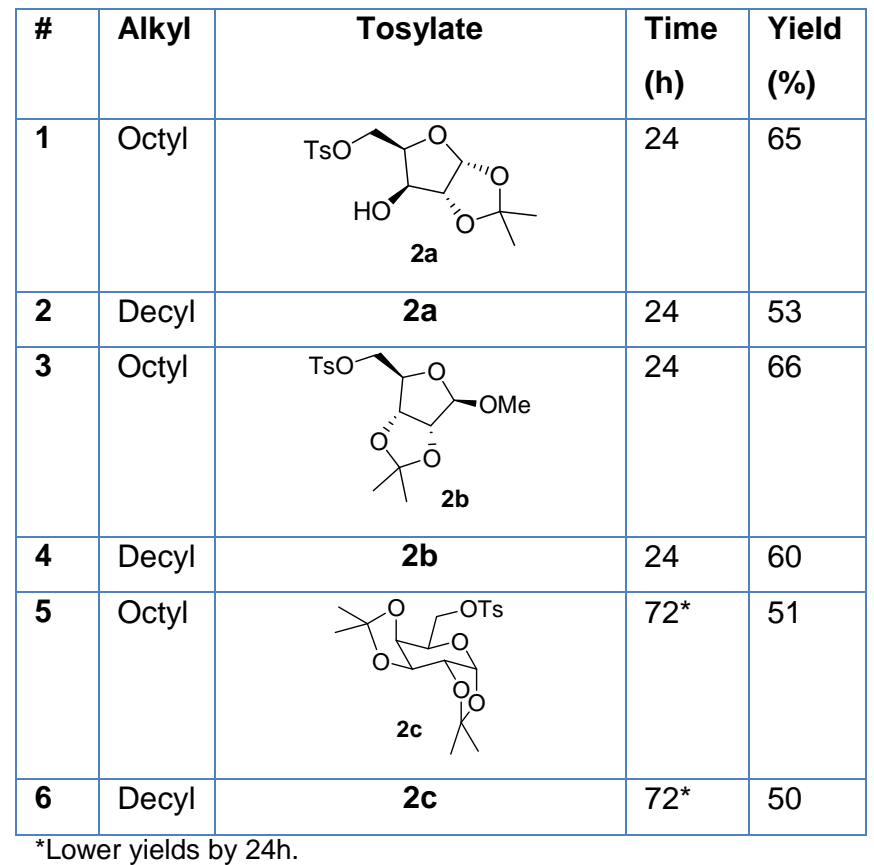

As we expected, the reaction carried out with octyl lead higher yields except for 2c, which is more hindered, the yields are modest and the reaction takes more time, 72 instead of 24 hours.

\section{CONCLUSION}

Here, we presented a useful and simple way to obtain a new class of molecules, the alkyl-selenoneoglycoconjugates. According to the previous works based on alkyl-glycosides, these molecules also showed potential biological activity and the antioxidant studies are in final adjustment course.

\section{ACKNOWLEDGEMENTS}

CAPES, CNPq, FAPERGS.

\section{REFERENCES}

${ }^{1}$ Boutureira, O.; Bernardes, G. J. L.; Fernandez-Gonzalez, M.; Anthony, D. C.; Davis, B. G. Angew. Chem. Int. Ed. 2012, 51, 1432.

${ }^{2}$ Matsumura, S., Imai, K., Yoshikawa, S., Kawada, K., Uchibori, T. J. Am. Oil Chem. Soc, 1990, 67, 996.

${ }^{3}$ May, S. W.; Pollock, S. H. Drugs 1998, 56, 959

${ }^{4}$ Affeldt, R. F.; Braga, H. C.; Baldassari, L. L.; Lüdtke, D. S. Tetrahedron, 2012, 68, 10470

${ }^{5}$ Rosevear, P.; VanAken,T; Baxter, J.; Ferguson-Miller, S. Biochemistry, 1980, 19, 4108. 